\title{
Obtención de Gelatina del Colageno de la Piel y Huesos del Cuy
}

\author{
Getting Gelatin Collagen Guinea Pig skin and Bones
}

\author{
Juan Orlando Chuquilin Arbildo', Gladys Vega Ventoncilla², Edwin Macavilca Ticlayauri ${ }^{2}$ \\ Fernando Eder Carreño Diaz², Máximo Rufino Mallqui ${ }^{2}$
}

\begin{abstract}
RESUMEN
Objetivo: Evaluar la técnica de extracción acido-base en la obtención de gelatina en carcasa de cuy y conocer el rendimiento del colágeno como una viabilidad tecnológica aceptable. Material y Métodos: Se emplearon cuyes tiernos (2,5 meses de edad) los cuales luego del sacrificio se obtuvo la carcasa, compuestas por la piel y la carne adherida a los huesos se empleó dos formas del material de obtención, que fueron comparados en su rendimiento, la primera compuesta por la piel y huesos del cuy, mientras que el segundo fue de la carcasa completa pero troceada (carne, piel y huesos), ambos fueron sometidos a un proceso de extracción acido-base con lavados sucesivos para la obtención de gelatina. Resultados: Las carcasas de cuy contienen colágeno, la comparación de rendimiento de gelatina a base de la piel y los huesos fue de $1900 \mathrm{~g}(25,5 \%)$, mientras que en la carcasa entera (carne, piel y huesos), se obtuvo $1133 \mathrm{~g}(6,6 \%)$ de concentrado de extracto gelatinoso. Conclusiones: La técnica de extracción acido-base propuesto para la obtención de gelatina a partir de las carcasas de cuy permitió obtener un producto aceptable, con escaso olor, color y textura. La técnica de extracción acido-base propuesto para la obtención de gelatina a partir de las carcasas de cuy permitió obtener con mayor rendimiento de gelatina a base de la piel y los huesos.
\end{abstract}

Palabras clave: Gelatina, colágeno, cuy, tratamiento acido-base

\begin{abstract}
Objective: To evaluate the acid-base extraction technique in obtaining gelatine in guinea pig carcass and to know the yield of collagen as an acceptable technological viability. Material and Methods: Young guinea pigs (2.5 months old) were used. After slaughter, the carcass was obtained, composed of the skin and meat adhered to the bones. Two forms of the obtained material were used, which were compared in Their performance, the first composed by the skin and bones of the guinea pig, while the second was of the complete but chopped carcass (meat, skin and bones), both were subjected to an acid-base extraction process with successive washes for the production Of gelatin. Results: Cuy carcasses contain collagen, the comparison of gelatin yield based on skin and bones was $1900 \mathrm{~g}(25.5 \%)$, whereas in the whole carcass (meat, skin and bones), Obtained $1133 \mathrm{~g}(6.6 \%)$ of gelatinous extract concentrate. Conclusions: The acid-base extraction technique proposed to obtain gelatine from guinea pig carcasses allowed an acceptable product to be obtained, with low odor, color and texture. The acid-base extraction technique proposed to obtain gelatine from the guinea pig shells allowed to obtain a higher gelatin yield based on skin and bones.
\end{abstract}

Keywords: gelatin, collagen, guinea pig, acid-base treatment

${ }_{1}^{1}$ Docente. Facultad de Ingeniería Química y Metalúrgica. Universidad Nacional José Faustino Sánchez Carrión. Email: jchuquilin123@hotmail.com.

${ }^{2}$ Docente. Facultad de Ingeniería Agraria, Industrias Alimentarias y Ambiental. Universidad Nacional José Faustino Sánchez Carrión. 


\section{INTRODUCCIÓN}

En los países andinos existe una población estable de más o menos 35 millones de cuyes. En el Perú, país con la mayor población y consumo de cuyes, se registra una producción anual de más de 16,500 toneladas de carne proveniente del beneficio de más de 65 millones de cuyes. Perú, el primer país productor de la carne de cuy y consumidor de la misma; el

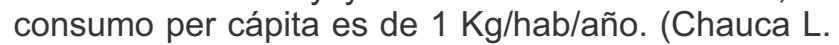
2003).

El cuy constituye un producto alimenticio de alto valor nutricional que en las últimas décadas se ha convertido en una carne requerida en el mercado nacional e internacional debido a su bajo nivel de grasas, su gran porcentaje en proteínas además de su exquisito sabor. La carne de cuy se caracteriza por ser una carne rica en proteínas $(20 \%)$ y a la vez pobre en grasas $(7 \%)$, ofreciendo una serie de beneficios nutricionales para quien lo consume. Presentación de la carne de cuy: entero en bolsa, entero en bandeja, entero sin patitas y cabeza, entero deshuesado, en mitad con cabeza y patitas, en mitad sin cabezas y patitas, piezado en brazuelos u piernas, y otros. (Chauca L. 2003).

La gelatina es un alimento natural y sano con una larga tradición. La gelatina es una proteína pura que se obtiene de materias primas animales que contienen colágeno. Este alimento natural y sano tiene un excelente poder de gelificar, pero eso no es todo, gracias a sus múltiples capacidades se emplea en los más diversos sectores industriales para un sin número de productos. (Lalja Mats y Larsson Mats 2004).

El colágeno es el tejido básico de la piel, los cartílagos y parte de los huesos. El colágeno es en sí muy poco soluble y, por ello, poco utilizado en alimentación. Cuando se convierte en gelatina cambian sus propiedades y aumenta el número de aplicaciones posibles. La fuente industrial de colágeno es generalmente las pieles $y$, a veces, los huesos de los diferentes animales. Estos productos se someten a cocción prolongada en agua hasta que el colágeno se va solubilizando y convirtiéndose en gelatina. A veces hay que acelerar el proceso acidificando o alcalinizando el agua. (Lalja Mats y Larsson Mats 2004).

En ese sentido, el presente trabajo consideró evaluar la técnica de extracción acido-base en la obtención de gelatina en la carcasa del cuy.

\section{MATERIALES Y MÉTODOS}

El trabajo experimental se realizó en el Laboratorio de procesos e ingeniería de alimentos de la Escuela Académica Profesional de Ingeniería de Industrias Alimentarias.

Materia prima: Se utilizó 100 cuyes jóvenes de $650 \mathrm{~g}$ de masa viva promedio, estos animales fueron sacrificados para obtener las carcasas (Piel, huesos, carne y patas) se cortó en trozos muy pequeños.

Pre tratamiento de las carcasas: La piel se enjuago con agua destilada (1:1) y se sometió a un pretratamiento acido base. Se mezcló con una solución
$\mathrm{NaOH}$ al $5 \%$ en proporción (1:1, piel: solución), se agito suavemente por 3 minutos y se dejó reposar durante 20 horas. Posteriormente se dreno la mezcla con una malla de $0,5 \mathrm{~mm}$ de diámetro y se agregó ácido acético $\left(\mathrm{CH}_{3} \mathrm{COOH}\right)$ al $5 \%$ (1:1, piel: solución).la mezcla se agito por 3 minutos y se dejó reposar durante 20 horas. Ambos procesos se realizaron a temperatura ambiente. A la piel fue drenada antes de someterse al proceso de extracción de sal. La piel tratada se adicionó una solución de $\mathrm{NaCl} 0,8 \mathrm{M}(1,1$, piel: solución) a $0{ }^{\circ} \mathrm{C}$ por 3 minutos, con cuatro repeticiones y se dreno después de cada lavado con malla de $0,5 \mathrm{~mm}$ de diámetro. Posteriormente la piel se mezcló con agua ( $10 \mathrm{~g}$ piel/ $/ 8 \mathrm{~mL}$ de agua destilada) y se mantuvo a $60{ }^{\circ} \mathrm{C} \times 60$ minutos con agitación constante. Finalmente se llevó a estufa en caja Petri a $50{ }^{\circ} \mathrm{C}$ por 24 horas. Obteniendo gelatina deshidratada.

Materiales y equipos: Pipetas, probetas, vasos de precipitados, colador, cuchara de madera, olla grande, estufa, PH-metro, termómetro, molino de martillos, decantador, balanza analítica.

Reactivos e insumos químicos: $\mathrm{NaOH} 5 \%$, ácido acético $\mathrm{CH}_{3}-\mathrm{COOH} 5 \%, \mathrm{NaCl}$

\section{Flujograma obtención de gelatina}

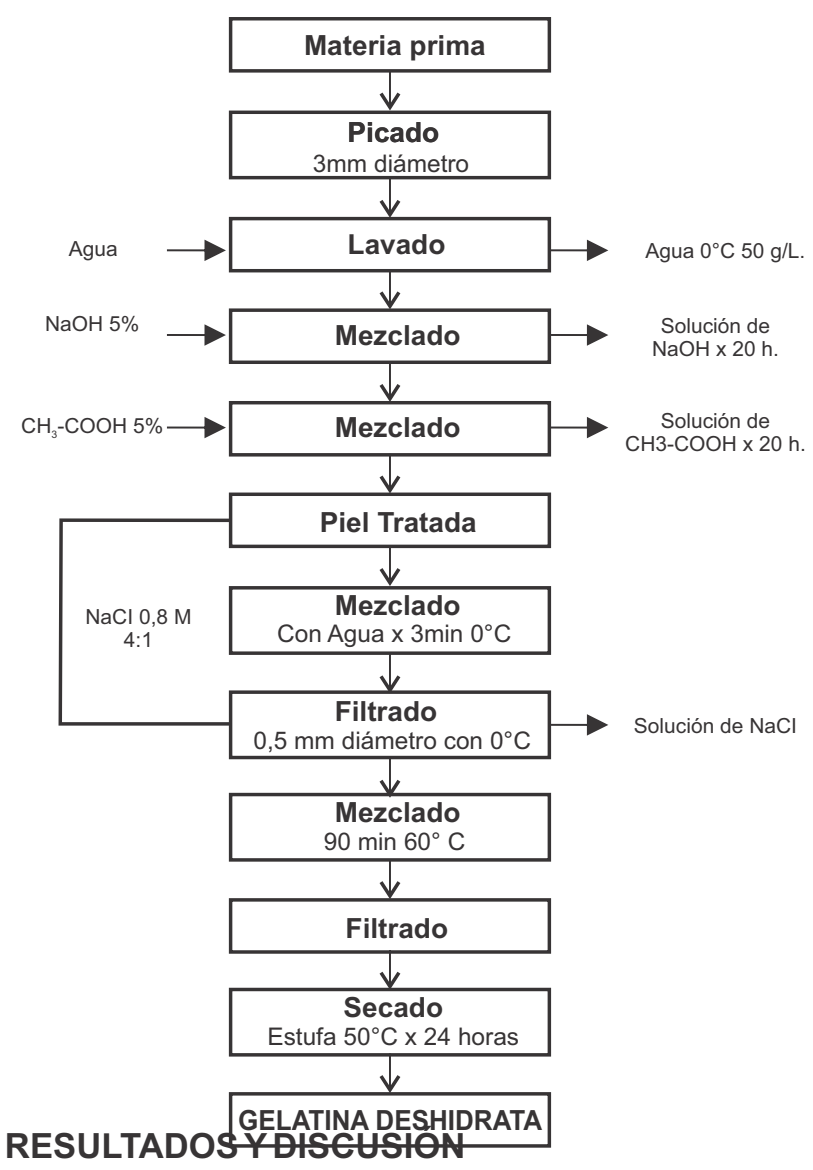

Se utilizó 100 cuyes en cada técnica. En la primera técnica se trabajó 50 cuyes donde se utilizó solo la piel y patas del animal, con una masa de $7450 \mathrm{~g}$, en la segunda técnica se trabajó 50 cuyes, donde se 
utilizó la carcasa entera del cuy es decir piel, carne y huesos, con un peso de $17250 \mathrm{~g}$. El resultado final del rendimiento de gelatina cruda extraída, obtenida del proceso modificado y usado en este caso para carcasa de cuy, se muestra en los cuadros 1 y 2 , donde señalan los gramos de gelatina extraída en cada uno de los procesos.

El rendimiento de gelatina a base de la piel y los huesos fue de $1900 \mathrm{~g}$, como se muestra en el Tabla 1.

El rendimiento de gelatina a base de la carcasa entera del cuy es decir piel, carne y huesos, fue de $1133 \mathrm{~g}$, como se muestra en el Tabla 2.

Tabla 1. Rendimiento de la Gelatina a Base de la Piel y Patas.

\begin{tabular}{ccc}
\hline Masa (g) & Solución de Gelatina (L) & Gelatina (g) \\
\hline 7450 & $\mathrm{NaOH} 5 \%$ y $\mathrm{CH}_{3}-\mathrm{COOH} 5 \%$ y NaCl 0,8 M & 1900 \\
\hline
\end{tabular}

Tabla 2. Rendimiento de la gelatina a base de la piel, carne y huesos.

\begin{tabular}{ccc}
\hline Masa (g) & Solución de Gelatina (L) & Gelatina (g) \\
\hline 17250 & $\mathrm{NaOH} 5 \%$ y $\mathrm{CH}_{3}-\mathrm{COOH} 5 \%$ y NaCl 0,8 M & 1133 \\
\hline
\end{tabular}

\section{CONCLUSIONES}

El método desarrollado para la obtención de gelatina de la carcasa del cuy, nos permitió obtener un producto aceptable con características de escaso olor, color y textura similar a la gelatina de patas y huesos de cerdos y vacunos.

La técnica de extracción acido-base propuesto para la obtención de gelatina a partir de la carcasa de cuy, permitió obtener un mayor rendimiento de gelatina a base de la piel y los huesos. Estos resultados indican el aprovechamiento comercial de la piel y huesos del cuy, sin embargo es necesario realizar mayores estudios e investigaciones para optimizar el proceso y mejorar la composición final del producto en polvo y su composición nutricional.

\section{AGRADECIMIENTOS}

Agradecemos a los docentes que prestan servicios en las facultades de Ingeniería Química y Metalúrgica y Ingeniería Agraria, Industrias Alimentarias y Ambiental por su colaboración desinteresada.

\section{REFERENCIAS BIBLIOGRÁFICAS}

Chauca L. (2003). Producción de cuyes. INIA. Lima. Perú.

Flores, I. (2001), Manual de Técnicas de laboratorio para la Industria Pecuaria. 1a ed. Edit. AASI, Riobamba, Ecuador. (pp 24-40).

Guadalupe R., Ramírez J. (2011). Extracción y caracterización de algunas propiedades fisicoquímicas de gelatina de piel de trucha. Universidad Autónoma de Tamaulipas. México.

Lalja Mats y Larsson Mats ((2004), Método para producir gelatina. Oficina española de patente y marcas. $N^{\circ}$ de public. 2122250 - Madrid. 